\title{
El estudio de casos como técnica de mejora docente en la asignatura de Taller de Arquitectura 6
}

\author{
Manuel Alejandro Pedreño Rojas \\ Universidad de Sevilla \\ Departamento de Construcciones \\ Arquitectónicas I \\ mpedreno@us.es \\ D.O.I.: http://dx.doi.org/10.12795/JDU.2018.i01.29 \\ Pp.: $517-530$
}

\section{Resumen}

Esta aportación pretende mostrar los resultados de aplicar un Ciclo de Mejora Docente (CMD) en la asignatura de Taller de Arquitectura 6 del Grado en Fundamentos de Arquitectura. Dicha asignatura se imparte en el segundo cuatrimestre del cuarto curso de la titulación. Posee un carácter multidisciplinar puesto que es impartida por seis profesores de diferentes disciplinas, que se agrupar para aportar su visión sobre un mismo proyecto de arquitectura que deben realizar los alumnos en grupos de un máximo de tres componentes. El descriptor de esta asignatura es la rehabilitación y por ello se les ha solicitado a los alumnos proponer una intervención en el antiguo Pósito de la Corredera de Córdoba como trabajo de curso. Teniendo en cuenta todos estos aspectos, el CMD propuesto pretende emplear la técnica del estudio de casos como herramienta para dotar a los estudiantes de referencias que les ayuden a abordar sus propios proyectos. 
Palabras Clave: Taller de Arquitectura 6; Grado en Fundamentos de Arquitectura; Docencia Universitaria; Experimentación Docente Universitaria; Estudio de Casos.

\section{Descripción del Contexto}

Con la llegada del Grado, se introduce en las titulaciones para obtener el título de Arquitecto las asignaturas de Taller de Arquitectura. Hasta ese momento, los estudiantes nunca se veían en la tesitura de integrar los conocimientos que aprendían en las diferentes asignaturas para la generación de un único proyecto. Este hecho suponía que el Proyecto Final de Carrera fuese la primera experiencia real integradora de todos estos conocimientos para diseñar un edificio o proyecto urbano. La asignatura de Taller de Arquitectura (6 ECTS) permanece constante a lo largo de toda la titulación desde segundo curso. En cada cuatrimestre posee un único descriptor común a todos los grupos, que sentará las bases sobre el tipo de proyecto que los alumnos deben realizar (ver Tabla 1). En concreto, el Taller de Arquitectura 6 (TA6) posee el Proyecto de Rehabilitación como descriptor común de la asignatura.

\section{Tabla 1}

Descriptor de las diferentes asignaturas de Taller de Arquitectura.

\begin{tabular}{lll}
\hline Asignatura & Curso & Descriptor \\
\hline Taller de Arquitectura 1 & 20 1C & Casa \\
\hline Taller de Arquitectura 2 & 20 2C & Bloque \\
\hline Taller de Arquitectura 3 & 30 1C & Equipamiento \\
\hline Taller de Arquitectura 4 & 3ㅇ 2C & Barrio \\
\hline Taller de Arquitectura 5 & 40 1C & Infraestructura \\
\hline Taller de Arquitectura 6 & 40 2C & Rehabilitación \\
\hline Taller de Arquitectura 7 & 50 1C & Ciudad \\
\hline & &
\end{tabular}

Jornadas de Formación e Innovación Docente del Profesorado | № 1 (2018) Esta obra se distribuye con la licencia Creative Commons Reconocimiento-NoComercial-SinObraDerivada Internacional (CC BY-NC-ND 4.0.) 
Debido al carácter multidisciplinar de la asignatura, en el TA6 imparten docencia seis profesores de las diferentes áreas de conocimiento de la Escuela (Proyectos Arquitectónicos, Construcción, Estructuras, Ingeniería del Terreno, Dibujo e Historia de la Arquitectura). Generalmente, salvo alguna excepción, los profesores que participan en el Taller son los mismos que imparten docencia simultáneamente al grupo de la asignatura que representan. En mi caso concreto, la asignatura de Construcción 5, en la cual se explican las diferentes técnicas y métodos de reparación de las lesiones que puedan aparecer en un edificio.

El desarrollo habitual de las clases de se basa en el concepto de "Taller". Los grupos de alumnos trabajan en el aula durante las 4 horas semanales de clase. Mientras, los docentes se pasan por las mesas realizando una corrección individualizada por los distintos grupos del trabajo. Los alumnos nos muestran sus avances y nos plantean sus dudas, colaborando el docente a la resolución de las mismas para conseguir al final del curso un resultado positivo materializado en un Proyecto de Arquitectura completo. Generalmente, salvo alguna excepción puntual, no se realizan clases teóricas. Únicamente se realizan dos sesiones críticas (una a mediados de cuatrimestre y otra al final) en la que los alumnos hacen públicos, tanto a los docentes como a sus compañeros, sus avances.

Condicionados por el descriptor de la asignatura, el grupo docente propone como trabajo de curso la intervención en el antiguo Pósito de la Corredera de Córdoba, dentro de un acurdo con la Gerencia de Urbanismo de la localidad. Se trata de un edificio de mediados del siglo XVI que actualmente se encuentra en un mal estado de conservación y para el cual el Ayuntamiento nos muestra su deseo de actuar, dándole un uso que le haga estar al servicio de la ciudadanía (ver Figura 1). 


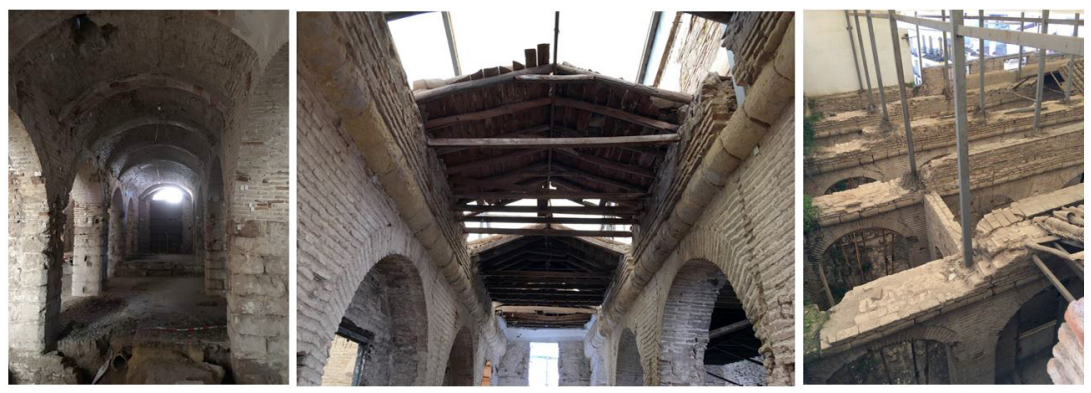

Figura 1. Estado actual del Pósito de la Corredera (Córdoba).

\section{Diseño previo del Ciclo de Mejora Docente}

Uno de los principales problemas que nos encontramos los arquitectos, y por consiguiente los estudiantes de arquitectura, es resolver constructiva y estructuralmente ciertos aspectos de nuestra idea original de proyecto. Este hecho tiene lugar debido a un planteamiento erróneo del proceso de proyectar. El arquitecto, a la vez que piensa su obra debe contemplar paralelamente otros aspectos fundamentales como son la materialidad deseada, el esquema estructural del edificio y su comportamiento, el planteamiento de ciertos detalles o encuentros constructivos, etc.

En este sentido, se ha elaborado el esquema de contenidos que se muestra en la Figura 2. En él se muestra la necesidad de aunar todos los conocimientos aportados por las diferentes disciplinas y ponerlos en práctica para la realización de un Proyecto de Rehabilitación.

Jornadas de Formación e Innovación Docente del Profesorado I № 1 (2018)

(c) Esta obra se distribuye con la licencia Creative Commons Reconocimiento-NoComercial-SinObraDerivada $\quad 4.0$ Internacional (CC BY-NC-ND 4.0.) 
TALLER DE ARQUITECTURA 6 (REHABILITACIÓN)

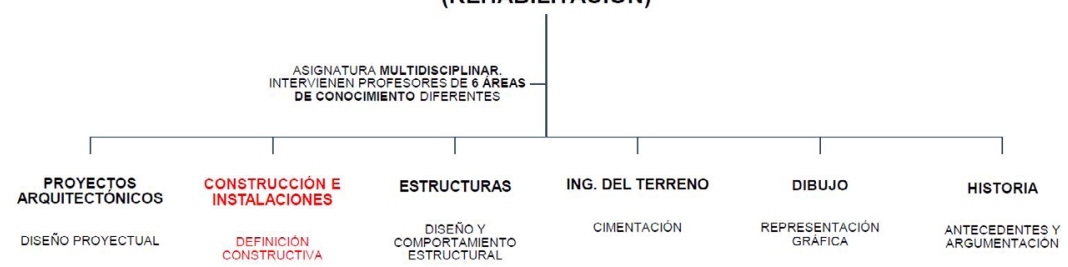

PROYECTO DE REHABILITACIÓN
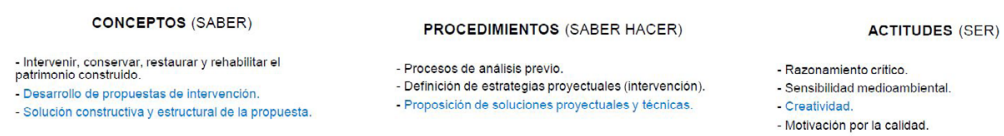

Figura 2. Esquema de contenidos elaborado para la asignatura de TA6.

Las actividades planteadas dentro del CMD (Porlán, 2017) pretenden ayudar al alumno a resolver y contemplar todos los aspectos que intervienen en un edificio a la vez que se va ideando el proyecto de la asignatura de taller. Para ello, cada grupo de trabajo, compuestos por un máximo 3 alumnos, analizará un proyecto de rehabilitación elegido por ellos. Lo ideal es que el estudio de este proyecto les ayude a resolver el trabajo de la asignatura, por lo es preferible que guarde relación con su idea de proyecto, al menos parcialmente.

El Ciclo de Mejora Docente se ha organizado en tres sesiones de clase (2 horas cada sesión) en las que programan las siguientes actividades:

- SESIÓN 1: se realizará en la sexta semana del cuatrimestre. Previamente se les habrá entregado a los estudiantes un enunciado con los objetivos perseguidos con la actividad. En esta sesión, cada grupo de alumnos preparará una pequeña presentación, de no más de 5 minutos, en la que deberán responder explícita o implícitamente a las siguientes cuestiones sobre el proyecto elegido: 
- ¿Por qué habéis elegido este proyecto? ¿Qué tiene de particular que os haya llamado la atención?

- Breve descripción del proyecto. ¿Cómo aborda el arquitecto la intervención en el edificio?

- ¿En qué ha consistido la rehabilitación del edificio? ¿Qué partes han sido sustituidas total o parcialmente?

- ¿Cómo es la materialidad del edificio? Breve descripción de sus fachadas y cubiertas (y otros elementos que se consideren interesantes) sin entrar en detalle. - ¿Cómo es la unión entre lo nuevo y lo preexistente?

Tras cada exposición se abrirá un breve turno de palabra en el que se destaquen los aspectos más útiles y relevantes del proyecto presentado. La jornada acabará con un debate de 15 minutos en el que los alumnos deberán extraer las conclusiones principales de la actividad y comenten su influencia en el trabajo del curso.

- SESIÓN 2: tendrá lugar la novena semana del cuatrimestre. En ella se generará un ambiente de trabajo a modo de workshop en el aula en el que cada grupo analizará un detalle concreto del edificio seleccionado realizando una definición completa y exhaustiva de materiales, uniones, comportamiento, etc. Este análisis les ayudará a transportar la solución constructiva estudiada a su proyecto de curso. Se plantean las siguientes actividades:

- Análisis de los detalles constructivos obtenidos de los edificios analizados (60 minutos).

- Breve exposición de los detalles estudiados por cada grupo para el resto de sus compañeros (45 minutos).

- Debate final y conclusiones (15 minutos).

- SESIÓN 3: se realizará la décima semana de curso y basará su funcionamiento en la trasposición, con las adaptaciones que sean necesaria, del detalle analizado en la segunda sesión al edificio que están diseñando para la asignatura de TA6. Se volverá a emplear 
la metodología de trabajo de workshop por grupos, trabajando cada uno de ellos en su propio proyecto. Mientras tanto, el docente se irá pasando por los distintos grupos para ayudarles a resolver las dudas que se le van planteando. El objetivo de la actividad no es que copien un determinado detalle, sino que reflexionen, apoyándose en lo ya estudiado, para dar una respuesta constructiva coherente a sus proyectos.

En la Figura 3 se muestra el modelo didáctico posible planteado para cada una de las sesiones del Ciclo de Mejora Docente.

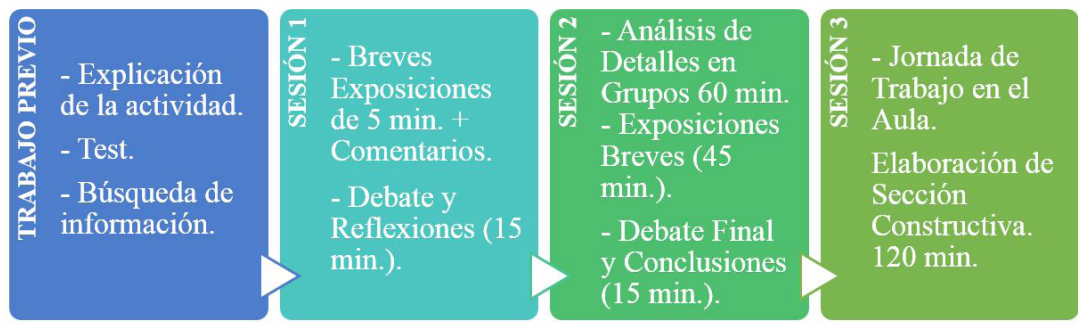

Figura 3. Modelo didáctico posible del CMD.

Con el objetivo de tener un seguimiento de la evolución de los alumnos, se les realiza un cuestionario previo a la realización del CMD. En él, los estudiantes individualmente deberán responder a los dos supuestos planteados.

- SUPUESTO 1: Te proponen participar como miembro del Jurado del Concurso para la intervención en el Antiguo Pósito de la Corredera de Córdoba. ¿Qué buscarías en un proyecto desde un punto de vista constructivo para hacerlo ganador? ¿Qué aspectos constructivos consideras fundamentales en una buena propuesta?

- SUPUESTO 2: Imagina que trabajas como Arquitecto Municipal del Ayuntamiento de Córdoba y debes dar el visto bueno a la propuesta ganadora del Concurso de Ideas para la Rehabilitación del Pósito de la Corredera. ¿Aceptarías cualquier proyecto? ¿Qué requisitos arquitectónicos, constructivos y formales le exigirías a la propuesta ganadora para darle el visado municipal? 


\section{Aplicación del Ciclo de Mejora Docente}

Tras el planteamiento de las tres sesiones que componen el CMD propuesto, a continuación se detalla la puesta en práctica de las jornadas antes definidas:

- SESIÓN 1: La clase anterior al ciclo de mejora se les solicita a los alumnos la preparación de una breve exposición de un proyecto de rehabilitación que les sea de interés. Disponen dos semanas para la preparación de la misma, enviándome vía email durante este periodo el edificio seleccionado.

Una vez conocida la lista de edificios analizados por los estudiantes, intento documentarme acerca de ellos para así poder ofrecer una mayor aportación por mi parte a la clase.

Antes del comienzo de la clase soy consciente que va a ser complicado que todos los grupos expongan sus trabajos ya que siempre las exposiciones suelen alargarse del tiempo preestablecido. Por ello, realizo una selección de los edificios por temáticas, priorizando aquellos proyectos que considero pueden ser de mayor interés para una mayor parte de alumnos.

Comenzamos la actividad con una breve introducción por mi parte en la que justifico la realización del ciclo y les explico el funcionamiento que tendrá la clase.

Comienzan a realizarse las exposiciones según lo previsto, observándose una importante demora en el tiempo cuando se tratan proyectos ya conocidos por los alumnos y que generan en ellos una gran curiosidad. Realizan muchas preguntas y plantean todas sus dudas sobre aspectos constructivos de las propuestas. 

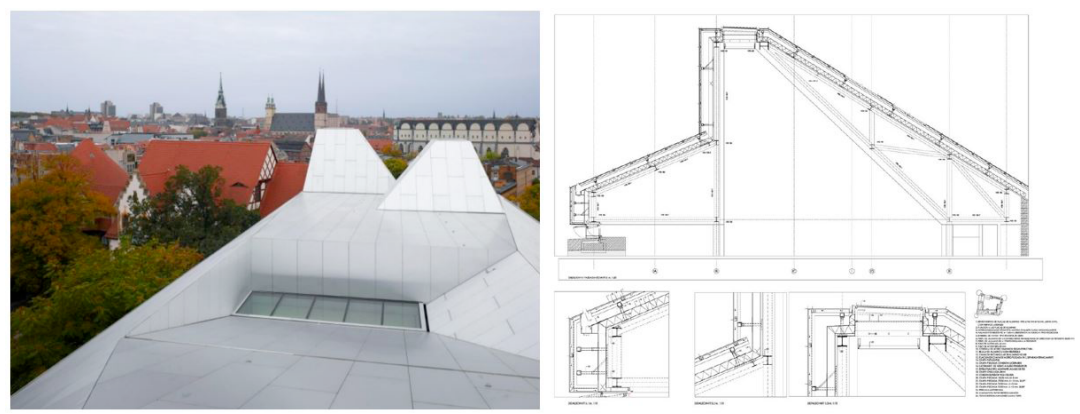

Figura 4. Ejemplo de edificio analizado por los estudiantes. Museo Moritzburg. Nieto y Sobejano.

Esa demora en los tiempos ha provocado que tan sólo diese tiempo a realizar cinco exposiciones, por lo que la planificación temporal previa por mi parte no ha sido correcta y es un aspecto importante a tener en cuenta en futuras actividades.

- SESIÓN 2: Al comienzo de la jornada los alumnos se distribuyen en el aula por grupos de trabajo, comenzando a analizar el detalle constructivo objeto de estudio. Esta actividad les lleva a analizar con profundidad el detalle constructivo. En él deben localizar y definir su materialidad, su estructura, su respeto con la edificación existente, su sensibilidad proyectual, etc.

Para realizar esta fase del CMD los alumnos han requerido más tiempo del previsto, necesitándose 75 minutos para realizar el análisis por grupo.
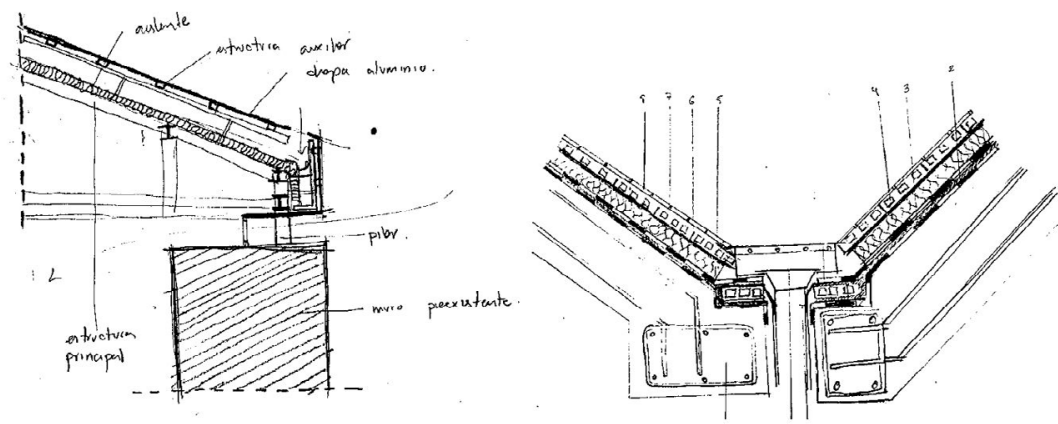

Figura 5. Ejemplo de análisis pormenorizado del detalle constructivo elaborado por los estudiantes.

Jornadas de Formación e Innovación Docente del Profesorado I № 1 (2018) Reconocimiento-NoComercial-SinObraDerivada Internacional (CC BY-NC-ND 4.0.) 
El hecho de aumentar el tiempo en la primera actividad de la sesión conlleva una reducción en el tiempo de exposición de los análisis. Por ello, se opta por reunir a todos los alumnos alrededor de una mesa y que los distintos grupos fuesen explicando los detalles que han analizado. Surge una conversación entre los alumnos muy enriquecedora de la que estoy seguro que han aprendido bastante.

La parte final de conclusiones se ve relegada a los últimos 5 minutos de clase. Por ello, me veo obligado a intervenir para comentar aquellos aspectos que he considerado de mayor relevancia para el grupo.

- SESIÓN 3: La tercera sesión se plantea como algo más privado en el propio grupo de trabajo. Una vez analizados los detalles del edificio modelo, los diferentes grupos intentaron poner en pie una primera versión de su sección constructiva durante las dos horas de clase.

Durante el desarrollo de la clase me ofrezco como ayuda a las dudas que les aparezcan a los estudiantes. En ningún caso pretendi darles la solución correcta (realmente no existe una única solución posible), sino ayudarles a que piensen una propuesta coherente y viable.

La evaluación de los cuestionarios previos realizados a los alumnos pretendía analizar su grado de sensibilidad al enfrentarse a un proyecto de rehabilitación de un edificio de carácter patrimonial como el que nos atañe. De la totalidad de respuestas recibidas, un 72 \% hace referencia únicamente a aspectos formales y espaciales del propio edificio. Una minoría, un $8 \%$, introduce también el concepto del análisis constructivo y material del edificio con el que se está trabajando. Este hecho se considera fundamental a la hora de enfrentarnos a un proyecto de estas características, ya que, frente a lo que sucede con las obras de nueva planta, la propuesta debe materializarse sobre 
un edificio existente, con unas características estructurales determinadas, que debe ser capaz de soportar aquello que se proyecta. Un reducido grupo, un $20 \%$ de los alumnos, contemplan, aparte de todo lo anterior, otro aspecto fundamental en este tipo de intervenciones. Introducen la importancia de considerar el entorno que rodea al edificio, ya que al ubicarse en un casco antiguo con una elevada protección urbanística hacen de éste un aspecto prioritario a la hora de pensar un buen proyecto en este enclave.

Con todo ello podemos afirmar que la escalera de aprendizaje previa de la clase se compone de tres niveles. En el inferior, y mayoritario, se encuentra el $72 \%$ del alumnado que únicamente fue capaz de identificar los aspectos más evidentes. El nivel intermedio lo componen dos alumnos (8 \% del total) los cuales entienden que es fundamental conocer la base con la que trabajamos. En el nivel superior se ubica el $20 \%$ del grupo, que aparte de lo anterior, identifica los valores urbanísticos y de entorno a tener en cuenta. Curiosamente, este último grupo se compone por los cinco alumnos que han ido llevando el ritmo del curso desde el primer día de clase, y que además mayor nivel interés han mostrado tanto por la actividad como por la asignatura.

Los resultados cosechados de esta actividad, como culmen de la totalidad del CMD, son bastante satisfactorios. Sin embargo, los detalles finales alcanzados por cada grupo difieren mucho los unos de los otros en función de la capacidad de trabajo y la dedicación de cada grupo. Se emplean esta primera versión de sección constructiva como cuestionario final del CMD. 


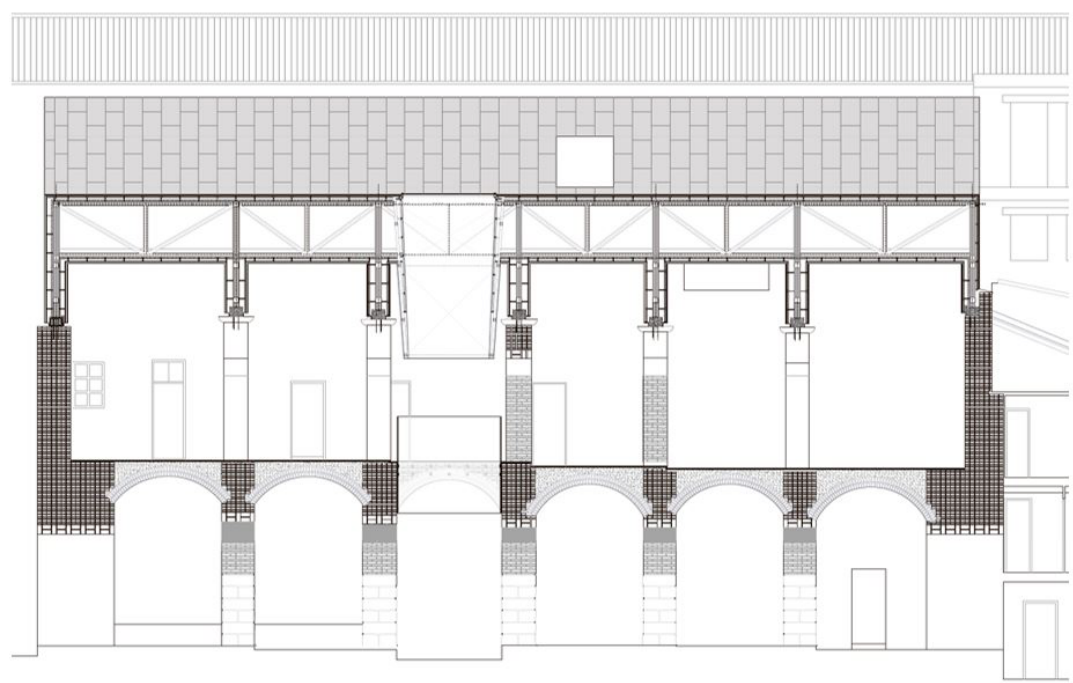

Figura 6. Ejemplo del resultado final alcanzado por los alumnos tras el CMD.

Del análisis de los detalles constructivos primitivos entregados por los estudiantes tras la última jornada se pueden establecer tres niveles o grupos en la clase. Existe un primer escalón, en el que se ubican dos grupos de trabajos, en el que los alumnos no han sido capaces de obtener los resultados deseados, bien sea por falta de interés o por falta de base previa. En el grupo intermedio se localizan cuatro grupos, los cuales has sido capaces de dar una solución constructiva más o menos coherente pero en la mayoría de los casos sin sensibilidad arquitectónica alguna. Finalmente, el nivel superior lo componen otros cuatro grupos que en este caso sí han sido capaces de utilizar el análisis previo para obtener un detalle coherente, bien ejecutado y sensible con su entorno y con el edificio sobre el que están trabajando.

Jornadas de Formación e Innovación Docente del Profesorado | № 1 (2018) Esta obra se distribuye con la licencia Creative Commons 
Tabla 2

Evaluación inicial y final de los conocimientos de los alumnos

\begin{tabular}{llc}
\hline \multicolumn{1}{c}{ Nivel } & Evaluación Inicial & Evaluación Final \\
\hline $\begin{array}{l}\text { Nivel } \mathbf{1} \\
\text { Únicamente identifican aspectos } \\
\text { evidentes }\end{array}$ & 18 alumnos (72\%) & 6 alumnos (24\%) \\
\hline $\begin{array}{l}\text { Nivel 2 } \\
\text { Entienden que es importante } \\
\text { conocer el edificio sobre el que } \\
\text { trabajan }\end{array}$ & 2 alumnos (8\%) & 10 alumnos (40\%) \\
\hline $\begin{array}{l}\text { Nivel 3 } \\
\begin{array}{l}\text { Además, identifican los valores } \\
\text { urbanísticos y del entorno. }\end{array}\end{array}$ & 5 alumnos (20\%) & 9 alumnos (36\%) \\
\hline
\end{tabular}

\section{Evaluación del Ciclo de Mejora Docente}

En líneas generales me encuentro bastante satisfecho con los resultados obtenidos tras la realización del CMD. Considero que buena parte de los alumnos han sabido utilizar lo aprendido en el estudio de casos para trasladarlo a sus propios proyectos.

En cuanto a la programación de las distintas sesiones, es cierto que resulta imposible explicar un edificio en 5 minutos. Este hecho obligó a que en la primera sesión muchos grupos no pudiesen exponer públicamente su análisis. En futuras ocasiones habrá que aumentar la duración de este tipo de sesiones expositivas.

Por otro lado considero que el estudio de casos debe ser una estrategia a implantar en todas las asignaturas de Taller de Arquitectura, pues al finalizar la misma los estudiantes poseen un amplio abanico de referencias sobre las que apoyarse para materializar sus proyectos.

Por último, destacar que una buena parte del alumnado me ha transmitido su satisfacción con la actividad realizada. Comentan que les ha sido de gran ayuda en muchos casos para saber resolver aspectos más técnicos de 
sus proyectos, para los cuales no contaban con la experiencia que en la vida real te da la práctica profesional.

\section{Conclusiones}

La realización del CMD desarrollado en esta comunicación pone de manifiesto la utilidad del "Estudio de Casos" (Finkel, 2008) como metodología de trabajo en asignaturas de carácter práctico. El hecho de que el alumno pueda de primera conocer qué han hecho otros anteriormente, para así solventar las dudas que se le originen, lo considero muy importante. Esto les otorga un mayor grado de independencia frente al profesor, ya que el ver otros ejemplos reales les hace crearse un criterio propio de aceptación que aquello que están realizando. Por otro lado, la puesta en común de ideas en clase, y el debate sobre las mismas, es una metodología docente atractiva para el alumno que, si se implica en la actividad, puede obtener un alto grado de conocimiento sobre un tema.

Ambas técnicas de trabajo las considero de gran utilidad y pretendo tomarlas como práctica habitual de mis clases a partir de este momento.

\section{Referencias Bibliográficas}

Bain, K. (2006). Lo que hacen los mejores profesores de universidad. Universitat de València.

Finkel, D. (2008). Dar clase con la boca cerrada. Universitat de València.

Porlán, R. et al. (2017). Enseñanza Universitaria. Cómo mejorarla. Editorial Morata.

Jornadas de Formación e Innovación Docente del Profesorado | № 1 (2018) Esta obra se distribuye con la licencia Creative Commons 